\title{
Simulation of adiabatic thermal beams in a periodic solenoidal magnetic focusing field
}

\author{
T. J. Barton, D. M. Field, K. M. Lang, and C. Chen \\ Plasma Science and Fusion Center, Massachusetts Institute of Technology, Cambridge, Massachusetts 02139, USA
}

(Received 27 March 2012; published 17 December 2012)

\begin{abstract}
Self-consistent particle-in-cell simulations are performed to verify earlier theoretical predictions of adiabatic thermal beams in a periodic solenoidal magnetic focusing field [K. R. Samokhvalova, J. Zhou, and C. Chen, Phys. Plasmas 14, 103102 (2007); J. Zhou, K. R. Samokhvalova, and C. Chen, Phys. Plasmas 15, 023102 (2008)]. In particular, results are obtained for adiabatic thermal beams that do not rotate in the Larmor frame. For such beams, the theoretical predictions of the rms beam envelope, the conservations of the rms thermal emittances, the adiabatic equation of state, and the Debye length are verified in the simulations. Furthermore, the adiabatic thermal beam is found be stable in the parameter regime where the simulations are performed.
\end{abstract}

DOI: 10.1103/PhysRevSTAB.15.124201

PACS numbers: 52.59.Sa, 05.10.-a, 05.20.-y, 29.27.-a

\section{INTRODUCTION}

Thermal beam equilibrium is desirable in highbrightness charged-particle beam systems where spacecharge effects play an important role in beam dynamics [1-3]. In particular, thermal beam equilibrium is a useful beam configuration, because there is no emittance growth if the beam is appropriately matched and if threedimensional effects are not important. It is also a useful beam configuration for minimizing beam loss. However, achieving thermal beam equilibrium in a practical system, in general, requires innovations in particle source design, beam matching, and system optimization, engineering and fabrication, especially for the injector of a particle accelerator where space-charge effects are often most significant. Indeed, few true thermal beam equilibria have been achieved in space-charge-dominated high-brightness beam systems, despite the growing needs of high-brightness beams in accelerators for x-ray free electron lasers, highenergy and nuclear physics research, and high-energydensity physics research.

The simplest example of thermal beam equilibrium is the rigid-rotor thermal beam equilibrium in a uniform magnet field [4-6]. This equilibrium describes a continuous charged-particle beam undergoing rigid rotation about its axis while propagating along the uniform magnet field. The temperature is isotropic in the beam frame in which the net momentum of the beam vanishes. It is a generalization of the Brillouin flow in which thermal effects are negligibly small [7]. When the beam is space-charge dominated, the equilibrium beam density is uniform in the beam core but drops off exponentially at the beam edge in a few Debye lengths. When the beam is emittance dominated, the

Published by the American Physical Society under the terms of the Creative Commons Attribution 3.0 License. Further distribution of this work must maintain attribution to the author(s) and the published article's title, journal citation, and DOI. thermal equilibrium beam density is Gaussian in the directions transverse to beam propagation. The distribution of the momentum relative to the fluid average is always Gaussian. Approximate rigid-rotor thermal beam equilibria were demonstrated experimentally [8], confirming certain theoretical predictions such as the beam focusing conditions and the angular velocity of beam rotation. They also revealed distortions in phase space.

Thermal beam equilibrium has been found theoretically in the smooth-focusing approximation for beam propagation in a periodic focusing field such as a periodic solenoidal magnetic focusing field or an alternating-gradient quadrupole magnetic focusing field [1-3]. For the periodic solenoidal magnetic field, the thermal beam equilibrium in the smooth-focusing approximation is essentially the rigidrotor thermal beam equilibrium in an equivalent uniform magnet field whose strength is equal to the rms average of the periodic solenoidal magnetic focusing field. For the alternating-gradient quadrupole magnetic focusing field, the thermal beam equilibrium in the smooth-focusing approximation describes a continuous charged-particle beam focused by an effective axisymmetric focusing potential that has a quadratic dependence on the transverse displacement. The density and momentum distributions of the thermal beam equilibrium in the smooth-focusing approximation are similar to the density and momentum distributions for the rigid-rotor thermal beam equilibrium in the Larmor frame. In general, the smooth-focusing approximation is valid when the vacuum phase advance is sufficiently small and the rms beam envelope is approximately a constant, as shown in perturbation theory $[9,10]$.

The thermal bunched-beam equilibrium has also been discussed in a moving, axisymmetric focusing field that provides both longitudinal focusing and axisymmetric transverse focusing for the beam [1]. In general, it describes an axisymmetric charged-particle bunch characterized by its rms beam radius and rms bunch length. The temperature is isotropic in the beam frame. When the 
bunch is space-charge dominated, the equilibrium beam density is a uniform density in the core of the bunch but drops off exponentially at the edge of the bunch in a few Debye lengths. When the bunch is emittance dominated, the equilibrium beam density is an axisymmetric Gaussian distribution. The thermal bunched-beam equilibrium is stable [9] inside a perfectly conducting circular beam tunnel.

Recently, the adiabatic thermal beam equilibrium has been predicted theoretically in a periodic solenoidal magnetic focusing field [11-13]. In general, an adiabatic thermal equilibrium corresponds to a spatially varying equilibrium state in the zero-order approximation of the Boltzmann equation. In the zero-order approximation, it is assumed that the distribution function is locally MaxwellBoltzmann, and that the density, temperature, average flow velocity, and self-electric and self-magnetic fields may vary in space. In particular, the existence of the adiabatic thermal beam equilibrium was shown in the framework of kinetic theory and equivalent warm-fluid theory. In the warm-fluid theory of the adiabatic thermal beam equilibrium [11,12], warm-fluid equations were solved in the paraxial approximation. The equation of state was adiabatic. The rms beam envelope, the density and flow velocity profiles, and the self-consistent Poisson equation were derived. In the kinetic theory of the adiabatic thermal beam equilibrium $[12,13]$, the thermal beam distribution function was constructed using the approximate and exact invariants of motion, i.e., a scaled transverse Hamiltonian and the angular momentum. By taking statistical averages, all of the equations in the warm-fluid theory were recovered, including the adiabatic equation of state, the rms beam envelope, the density and flow velocity profiles, and the self-consistent Poisson equation.

It was shown in a numerical study that chaotic particle motion is almost completely eliminated in the adiabatic thermal beam equilibrium [14]. The importance of this result is twofold: First, the elimination of chaotic particle motion provides a further numerical proof that the scaled transverse Hamiltonian is a very good approximate constant of motion. This approximate constant of motion and the exact constant of motion of the canonical angular momentum assure that the motion of charged particles is approximately integrable in the four-dimensional phase space of the adiabatic thermal beam equilibrium. Second, the elimination of chaotic particle motion may provide valuable insight into how to control chaotic particle motion, halo formation, and beam loss in beam transport channels. In particular, if adiabatic thermal beams are stable, they may be used to control chaotic particle motion, beam halo formation, and beam loss.

Some evidence was found that the theoretical prediction of the beam density is supported by the experimental data from a high-intensity beam experiment at the University of Maryland Electron Ring (UMER) [15]. However, agreement between the theory and the experiment was found only for a short propagation distance that was less than one focusing period $[12,13]$. The lack of agreement between the theory and the experiment may be due to the fact that the beam was not created under the conditions required for the thermal beam equilibrium. Indeed, thermal beam equilibrium generation is still an important topic of research in beam physics.

The discovery of the adiabatic thermal beam equilibrium was an important advance in beam physics, overcoming the shortcoming of the Kapchinskij-Vladmirskij (KV)-type beam equilibrium in a periodic solenoidal magnetic focusing field $[1-4,9,16,17]$. The KV-type beam equilibrium has a singular ( $\delta$-function) distribution in the four-dimensional phase space. Such a $\delta$-function distribution gives a uniform density profile across the beam in the transverse directions, and a transverse temperature profile which peaks on axis and decreases in a quadratic manner to zero on the edge of the beam. Because of the singularity in the distribution functions, KV beam equilibria are not likely to occur in real physical systems and cannot provide realistic models for theoretical and experimental studies and simulations except in the zero-temperature limit. In KV beam equilibria, there is no relation between temperature and density, and therefore, there is no equation of state. For example, the KVtype beam equilibrium model cannot be used to explain the beam tails in the radial distributions observed in the UMER experiment [15]. Furthermore, if one attempts to describe a realistic beam using the concept of an equivalent $\mathrm{KV}$ beam [1], the quadratic temperature profile induces an artificially repulsive pressure force, resulting in not only an unrealistically lower density of the beam on the axis but also unrealistic beam dynamics in phase space [15].

In this paper, we report results of two-dimensional (2D) particle-in-cell (PIC) simulations which further validate the theoretical predictions of the adiabatic thermal beam equilibrium [11,13]. In particular, we discuss simulation results for adiabatic thermal beams that do not rotate in the Larmor frame. For such beams, the simulation results verify the theoretical predictions of the rms beam envelope, the conservations of the rms thermal emittances, the adiabatic equation of state, and the Debye length. They also show beam stability in the parameter regime where the simulations are performed.

The organization of the present paper is as follows. In Sec. II, we discuss the 2D PIC model for adiabatic thermal beams in a periodic solenoidal magnetic focusing field, and describe the initialization of an adiabatic thermal beam. In Sec. III, we discuss results of 2D PIC simulations, compare them with theoretical predictions, and find good agreement between simulation and theory. In Sec. IV, we present the conclusion and discuss directions for future investigation.

\section{PARTICLE-IN-CELL MODEL}

We study self-consistent charged-particle dynamics in the adiabatic thermal equilibrium of an intense charged-particle 
beam propagating with constant axial velocity $\beta_{b} c \hat{\mathbf{e}}_{z}$ in the periodic solenoidal magnetic focusing field,

$$
\mathbf{B}^{\mathrm{ext}}=B_{z}(s) \hat{\mathbf{e}}_{z}-\frac{1}{2} \frac{d B_{z}(s)}{d s}\left(x \hat{\mathbf{e}}_{x}+y \hat{\mathbf{e}}_{y}\right),
$$

where $s=z$ is the axial coordinate, $B_{z}(s+S)=B_{z}(s)$ is the axial magnetic field, $S$ is the fundamental periodicity length of the focusing field, and $c$ is the speed of light in vacuum.

The paraxial approximation is made under the following assumptions: (1) $r_{b r m s} \ll S$, where $r_{b r m s}$ is the rms beam radius, and (2) $\nu / \gamma_{b}^{3} \beta_{b}^{2} \ll 1$, where $\nu=q^{2} N_{b} / m c^{2}$ is the Budker parameter of the beam, $q$ and $m$ are the particle charge and rest mass, respectively, $N_{b}=$ $\int_{0}^{\infty} n_{b}(r, s) 2 \pi r d r=$ const is the number of charged particles per unit axial length, and $\gamma_{b}=\left(1-\beta_{b}^{2}\right)^{-1 / 2}$ is the relativistic mass factor.

The basic equations in the 2D PIC model are expressed in cgs units as

$$
\begin{gathered}
\frac{d^{2} \tilde{\mathbf{x}}_{\perp i}}{d s^{2}}+\kappa_{z}(s) \tilde{\mathbf{x}}_{\perp i}+\frac{q}{\gamma_{b}^{3} m \beta_{b}^{2} c^{2}} \frac{\partial \phi\left(\tilde{\mathbf{x}}_{\perp i}, s\right)}{\partial \tilde{\mathbf{x}}_{\perp i}}=0, \\
\frac{\partial^{2} \phi\left(\tilde{\mathbf{x}}_{\perp i}, s\right)}{\partial \tilde{\mathbf{x}}_{\perp i}^{2}}=-4 \pi q n_{b}\left(\tilde{\mathbf{x}}_{\perp i}, s\right),
\end{gathered}
$$

where $i=1,2, \ldots, N_{P}, \sqrt{\kappa_{z}(s)}=q B_{z}(s) / 2 \gamma_{b} m \beta_{b} c^{2}, \phi$ is the electrostatic potential, $n_{b}$ is the beam density, and $\tilde{\mathbf{x}}_{\perp i}$ is the transverse position of the $i$ th macroparticle in the Larmor frame, i.e., $\tilde{x}=x \cos \varphi-y \sin \varphi$ and $\tilde{y}=x \sin \varphi+$ $y \cos \varphi$ with $\varphi=\int_{0}^{s} \sqrt{\kappa_{z}(s)} d s$.

In the 2D PIC simulations, the focusing field is chosen to be sinusoidal with $S \sqrt{\kappa_{z}(s)}=(2 / 3)^{1 / 2} \sigma_{0}[1+$ $\cos (2 \pi s / S)]$, where $\sigma_{0}$ is the vacuum phase advance. Poisson's equation (3) is solved using a successive over relaxation algorithm in a grounded circular perfect conducting pipe of radius $R$ centered on the $z$ axis on a square mesh. The value of $R$ is chosen to be several times that of the maximum value of the rms beam radius.

The 2D PIC simulations discussed in this paper are limited to a class of adiabatic thermal beams that do not rotate in the Larmor frame. For such a class of beams, the macroparticles are loaded at $s=0$ according to the initial distribution function $[12,13]$

$$
f\left(\tilde{\mathbf{x}}_{\perp}, \tilde{\mathbf{x}}_{\perp}^{\prime}, s=0\right)=n_{b}(\tilde{r}, 0) \exp \left\{-\frac{\gamma_{b} m \beta_{b}^{2} \tilde{\mathbf{x}}_{\perp}^{\prime 2}}{2 k_{B} T_{\perp}(0)}\right\}
$$

where $\tilde{r}=\left(\tilde{x}^{2}+\tilde{y}^{2}\right)^{1 / 2}$, prime denotes derivative with respect to $s, k_{B}$ is the Boltzmann constant, and $T_{\perp}(0)$ and $n_{b}(\tilde{r}, 0)$ are the initial beam temperature and density, respectively. The density $n_{b}(\tilde{r}, 0)$ is given by

$$
n_{b}(\tilde{r}, 0)=\frac{4 \pi C \varepsilon_{t h}^{2}}{r_{b r m s}^{2}(0)} \exp \left\{-\frac{K \tilde{r}^{2}}{8 \varepsilon_{t h}^{2}}-\frac{\tilde{r}^{2}}{r_{b r m s}^{2}(0)}-\frac{q \phi(\tilde{r}, 0)}{\gamma_{b}^{2} k_{B} T_{\perp}(0)}\right\}
$$

where $C$ is a constant determined by $N_{b}=$ $\int_{0}^{\infty} n_{b}(\tilde{r}, s) 2 \pi \tilde{r} d \tilde{r}, K \equiv 2 q^{2} N_{b} / \gamma_{b}^{3} m \beta_{b}^{2} c^{2}$ is the generalized beam perveance, the initial electrostatic potential $\phi(\tilde{r}, 0)$ is determined from the Poisson equation

$$
\frac{1}{\tilde{r}} \frac{\partial}{\partial \tilde{r}}\left(\tilde{r} \frac{\partial}{\partial \tilde{r}}\right) \phi(\tilde{r}, 0)=-4 \pi q n_{b}(\tilde{r}, 0),
$$

and $r_{b r m s}(0)$ and $r_{b r m s}^{\prime}(0)=0$ are the initial conditions of the periodic rms beam envelope $r_{\text {brms }}(s)=r_{\text {brms }}(s+S)$ that solves the rms beam envelope equation $[12,13]$ :

$$
\frac{d^{2} r_{b r m s}}{d s^{2}}+\kappa_{z}(s) r_{b r m s}-\frac{K}{2 r_{b r m s}}=\frac{4 \varepsilon_{t h}^{2}}{r_{b r m s}^{3}} .
$$

The trajectories of the macroparticles are integrated using the standard leapfrog method. The 2D PIC algorithm is implemented in a MATLAB version of the MIT 2D periodically focused beam (PFB2D) code which was used extensively in simulation studies of high-brightness charged-particle beams in periodic focusing fields [18].

\section{SIMULATION RESULTS}

Two-dimensional PIC simulations were performed to verify the theoretical predictions of the following [11-13]: (a) rms beam envelope; (b) conservation of rms thermal emittances; (c) adiabatic equation of state; (d) density, velocity, and temperature profiles; and (e) Debye length. Results of a typical simulation are shown in Figs. $1-4$ for the parameters: $S^{2} \kappa_{z}(s)=(2 / 3) \sigma_{0}^{2}[1+$ $\cos (2 \pi s / S)]^{2}, \sigma_{0}=80^{\circ}, S K / 4 \varepsilon_{t h}=7.0, N_{P}=1 \times 10^{6}$, $\left(4 \varepsilon_{t h} S\right)^{-1 / 2} R=5.0$, integration step size $\Delta s / S=0.01$, and mesh size $\left(4 \varepsilon_{t h} S\right)^{-1 / 2} \Delta \tilde{x}=0.02$.

The normalized rms beam radius $\left(4 \varepsilon_{t h} S\right)^{-1 / 2} r_{b r m s}$ from the simulation is plotted in Fig. 1 as a function of the normalized distance $s / S$. Within the statistical error

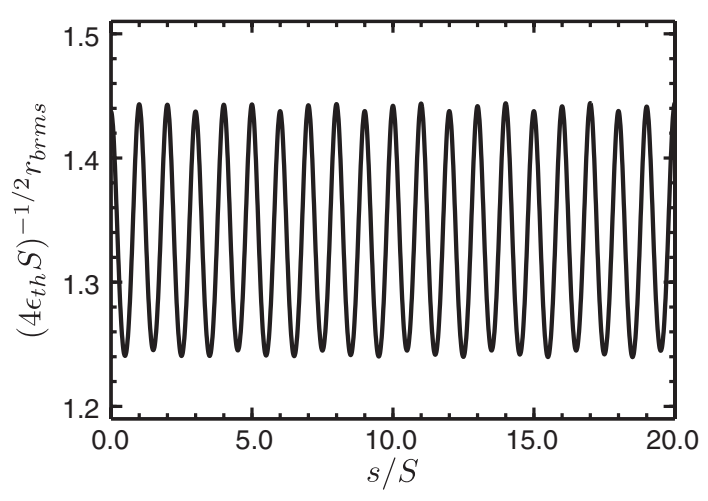

FIG. 1. Plot of the normalized rms beam radius $\left(4 \varepsilon_{t h} S\right)^{-1 / 2} r_{b r m s}$ versus the normalized distance $s / S$. 



FIG. 2. Plots of the probability density distributions $\rho\left(\tilde{x}^{\prime}\right)$ versus $\left(S / 4 \varepsilon_{t h}\right)^{1 / 2} \tilde{x}^{\prime}$ and the probability density distributions $\rho\left(\tilde{y}^{\prime}\right)$ versus $\left(S / 4 \varepsilon_{t h}\right)^{1 / 2} \tilde{y}^{\prime}$ at $s / S=20$. The dotted curves are from the simulation, whereas the dashed curves are from the theoretical predictions.

of $\pm 1 \%$, the result agrees with the matched beam envelope solution of Eq. (7).

The transverse momentum distribution continues to be Gaussian, as illustrated in Fig. 2. The beam density distribution maintains a plateau distribution as shown in Fig. 3 in which the normalized beam density $\left(4 \varepsilon_{t h} S / N_{b}\right) n_{b}$ is plotted as a function of the normalized radius $\left(4 \varepsilon_{t h} S\right)^{-1 / 2} r$ at $s / S=20$. The dotted curves are from the simulation, whereas the dashed curves are from the theoretical predictions. The characteristic scale over which the beam density

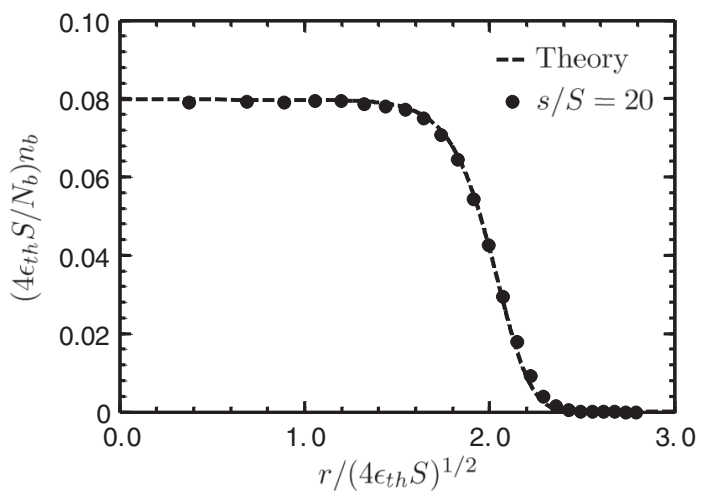

FIG. 3. Plot of the normalized beam density $\left(4 \varepsilon_{t h} S / N_{b}\right) n_{b}$ versus the normalized radius $\left(4 \varepsilon_{t h} S\right)^{-1 / 2} r$ at $s / S=20$.



FIG. 4. Plots of the relative rms thermal emittances $\varepsilon_{\tilde{x} r m s} / \varepsilon_{\tilde{x} r m s}(0)$ and $\varepsilon_{\tilde{y} r m s} / \varepsilon_{\tilde{y} r m s}(0)$ versus the normalized distance $s / S$.

falls is the Debye length $\lambda_{D}$, which, in this example, has a normalized value of $\left(4 \varepsilon_{t h} S\right)^{-1 / 2} \lambda_{D}=0.14$. There is good agreement between the simulation and theory.

In Fig. 4, the evolutions of the relative rms thermal emittances $\varepsilon_{\tilde{x} r m s} / \varepsilon_{\tilde{x} r m s}(0)$ and $\varepsilon_{\tilde{y} r m s} / \varepsilon_{\tilde{y} r m s}(0)$ in the simulation are shown in solid and dashed curves, respectively, as a function of the normalized distance $s / S$. Here, $\varepsilon_{\tilde{x} r m s}(0)$ and $\varepsilon_{\tilde{y} r m s}(0)$ are the rms emittances in the $\tilde{x}$ and $\tilde{y}$ directions at $s=0$, respectively. Both rms thermal emittances are conserved within the statistical error of $\pm 0.3 \%$. These results are in agreement with the theoretical prediction of the conservation of rms thermal emittances, and validate the adiabatic equation of state [11,12].

In the parameter regimes where simulation studies were performed, we did not find any indication of beam instability. The results shown in Figs. 1-4 are typical; that is, the Gaussian transverse momentum distributions, the plateau beam density distribution, the temperature, and rms beam radius repeat themselves as the beam propagates through every period in the focusing field.

While a complete proof of the stability of adiabatic thermal beams is not available, the present simulations are consistent with the previously reported simulations showing that a beam with a semi-Gaussian distribution (i.e., a distribution that is Gaussian in the momentum space and uniform in the configuration space) suffers from considerably less emittance growth than a beam with a KV distribution [19].

\section{CONCLUSION}

Two-dimensional particle-in-cell simulations were performed to verify the earlier theoretical predictions of adiabatic thermal beams in a periodic solenoidal magnetic focusing field. Results were obtained for adiabatic thermal beams that do not rotate in the Larmor frame. For such beams, the theoretical predictions of the rms beam envelope, the conservations of the rms thermal emittances, the adiabatic equation of state, and the Debye length were verified in the $2 \mathrm{D}$ PIC simulations. Furthermore, the 
adiabatic thermal beam was found to be stable in the parameter regime where the simulations were performed.

The generation of an adiabatic thermal beam in a practical beam device will be an important topic of future research in beam physics. In general, achieving thermal beam equilibrium in a practical system will still require innovations in particle source design, beam matching, and system optimization, engineering and fabrication, especially for the beam injector where space-charge effects are most significant.

\section{ACKNOWLEDGMENTS}

This research was supported by DOE Grant No. DEFG02-95ER40919 and Grant No. DE-FG02-05ER54836 and MIT Undergraduate Research Opportunity Program (UROP).

[1] M. Reiser, Theory and Design of Charged-Particle Beams (Wiley, New York, 1994), Chap. 5.

[2] J.D. Lawson, The Physics of Charged-Particle Beams (Clarendon, Oxford, 1988).

[3] T.P. Wangler, Principles of $R F$ Linear Accelerators (Wiley, New York, 1998).

[4] R. C. Davidson, Physics of Nonneutral Plasmas (AddisonWesley, Redwood City, CA, 1990), p. 52.

[5] R. C. Davidson and N. A. Krall, Phys. Rev. Lett. 22, 833 (1969).
[6] R.C. Davidson and H.S. Uhm, Phys. Fluids 22, 1375 (1979).

[7] L. Brillouin, Phys. Rev. 67, 260 (1945).

[8] A. J. Theiss, R. A. Mahaffey, and A. W. Trivelpiece, Phys. Rev. Lett. 35, 1436 (1975).

[9] R. C. Davidson and H. Qin, Physics of Intense Charged Particle Beams in High Energy Accelerators (Imperial College Press, Singapore, 2001).

[10] R. C. Davidson, H. Qin, and P. J. Channell, Phys. Rev. ST Accel. Beams 2, 074401 (1999); Phys. Lett. A 258, 297 (1999).

[11] K. R. Samokhvalova, J. Zhou, and C. Chen, Phys. Plasmas 14, 103102 (2007).

[12] K. R. Samokhvalova, Ph.D. thesis, Massachusetts Institute of Technology, 2008.

[13] J. Zhou, K. R. Samokhvalova, and C. Chen, Phys. Plasmas 15, 023102 (2008).

[14] H. Wei and C. Chen, Phys. Rev. ST Accel. Beams 14, 024201 (2011).

[15] S. Bernal, B. Quinn, M. Reiser, and P. G. O'Shea, Phys. Rev. ST Accel. Beams 5, 064202 (2002).

[16] I. M. Kapchinskij and V.V. Vladimirskij, in Proceedings of the Conference on High Energy Accelerators (CERN, Geneva, 1959), p. 274.

[17] C. Chen, R. Pakter, and R. C. Davidson, Phys. Rev. Lett. 79, 225 (1997).

[18] J. Zhou, R. Bhatt, and C. Chen, Phys. Rev. ST Accel. Beams 9, 034401 (2006).

[19] J. Struckmeier, J. Klabounde, and M. Reiser, Part. Accel. 15, 47 (1984). 